\title{
Phosphorylation of estrogen receptor $\alpha$ at serine 118 is correlated with breast cancer resistance to tamoxifen
}

\author{
MING CHEN ${ }^{1,2}$, YU-KUN CUI ${ }^{3}$, WEN-HE HUANG ${ }^{1}$, KWAN MAN $^{4}$ and GUO-JUN ZHANG ${ }^{1,5}$ \\ ${ }^{1}$ Breast Center of The Affiliated Cancer Hospital of Shantou University Medical College, Shantou, Guangdong 515031; \\ ${ }^{2}$ Department of Obstetrics and Gynecology, Peking Union Medical College Hospital, \\ Chinese Academy of Medical Sciences and Peking Union Medical College, Beijing 100730; \\ ${ }^{3}$ The Central Laboratory of The Affiliated Cancer Hospital of Shantou University Medical College, Shantou, \\ Guangdong 515031; ${ }^{4}$ Department of Surgery, Li Ka-Shing Faculty of Medicine, Hong Kong University, Hong Kong, SAR; \\ ${ }^{5}$ Cancer Research Center, Shantou University Medical College, Shantou, Guangdong 515041, P.R. China
}

Received December 24, 2012; Accepted April 9, 2013

DOI: $10.3892 / \mathrm{ol} .2013 .1324$

\begin{abstract}
The aim of the present study was to explore the correlation between estrogen receptor $\alpha(E R \alpha)$ phosphorylation at serines 118 and 167 and the responsiveness of patients with primary breast cancer to tamoxifen. Tumors from 104 patients with primary breast cancer who received adjuvant tamoxifen therapy at The Affiliated Cancer Hospital of Shantou University Medical College between January 2001 to December 2007 were subjected to immunohistochemical analysis with specific antibodies against ER $\alpha$ phosphorylated at either serine 118 (pER $\alpha-S 118)$ and/or serine 167 (pER $\alpha-S 167)$. $\mathrm{ER} \alpha$ phosphorylation at the two sites was correlated with either the disease-free survival or the overall survival rate of these patients using the Kaplan-Meier survival analysis. pER $\alpha-S 118$ and $\mathrm{pER} \alpha-\mathrm{S} 167$ were found to be expressed in the cell nucleus of $25.0 \%(26 / 104)$ and $26.9 \%(28 / 104)$ of breast cancers, respectively. The expression of $\mathrm{pER} \alpha-\mathrm{S} 118$ was positively correlated with the human epidermal growth factor receptor-2 (HER-2) status $\left(\chi^{2}=6.85, \mathrm{P}=0.01\right)$. The Kaplan-Meier analysis revealed a poorer disease-free $(\mathrm{P}=0.022)$ and overall survival $(\mathrm{P}=0.013)$ in breast cancer patients expressing $\mathrm{pER} \alpha-\mathrm{S} 118$, but not in those expressing $\mathrm{pER} \alpha-\mathrm{S} 167$. In conclusion, $\mathrm{pER} \alpha-\mathrm{S} 118$ was correlated with the HER-2 status and predicted breast cancer resistance to tamoxifen.
\end{abstract}

\section{Introduction}

Breast cancer is the most frequently detected female neoplasm worldwide. The estrogen receptor (ER) is expressed by $60-70 \%$

Correspondence to: Professor Guo-Jun Zhang, Breast Center of The Affiliated Cancer Hospital of Shantou University Medical College, 7 Raoping Road, Shantou, Guangdong 515031, P.R. China E-mail: guoj_zhang@yahoo.com

Key words: estrogen receptor $\alpha$, phosphorylation, tamoxifen, breast cancer, endocrine resistance of breast tumors (1); therefore, targeting the ER has emerged as a major management technique for ER-positive breast cancers. Tamoxifen is the most commonly used antiestrogen, but resistance remains the major obstacle for its clinical application. While up to one-third of patients are resistant to tamoxifen at the beginning of treatment, the majority of patients who initially respond to tamoxifen will later also become resistant (2). Although the mechanisms underlying tamoxifen resistance are largely unknown, increasing evidence has indicated that $\mathrm{ER} \alpha$ cross-communicates with growth factor signaling, including epidermal growth factor receptor (EGFR) and mammalian target of rapamycin (mTOR) signaling, and the resultant ER $\alpha$ phosphorylation, are important in tamoxifen resistance (3).

As a ligand-activating transcription factor, ER $\alpha$ has been found to be phosphorylated at numerous sites, including serines 118, 167, and 305 (4). These three sites have been demonstrated to be phosphorylated by extracellular signal-regulated kinase/mitogen-activated protein kinase (ERK/MAPK), protein kinase B (Akt) and protein kinase A (PKA) and/or p21-activated protein kinase (Pak1), respectively, and appear to be the most relevant sites with regard to breast cancer resistance to tamoxifen (4). Certain studies have demonstrated that the reduced ER $\alpha$ phosphorylation at serine 118 (pER $\alpha-S 118)$ and the increased ER $\alpha$ phosphorylation at serine 167 ( $\mathrm{pER} \alpha-\mathrm{S} 167)$ were correlated significantly with the improved disease-free survival (DFS) and overall survival (OS) of breast cancer patients, while alternative studies have suggested a correlation between either $\mathrm{pER} \alpha-\mathrm{S} 118$ or $\mathrm{pER} \alpha-\mathrm{S} 167$ and tamoxifen resistance (5). The effects of pER $\alpha-S 118$ and pER $\alpha-S 167$ have not yet been elucidated, and therefore further studies are required.

In the present study, the status of $\mathrm{pER} \alpha-\mathrm{S} 118$ and $\mathrm{pER} \alpha-$ S167 proteins were immunohistochemically detected in breast tumors from patients who received adjuvant tamoxifen treatment, and the clinicopathological features, the DFS and the OS of these patients were correlated with the status of either $\mathrm{pER} \alpha-\mathrm{S} 118$ or $\mathrm{pER} \alpha-\mathrm{S} 167$. The results showed that ER $\alpha$ phosphorylation was correlated with human epidermal growth factor receptor-2 (HER 2) status at serine (Ser) 118, but not 

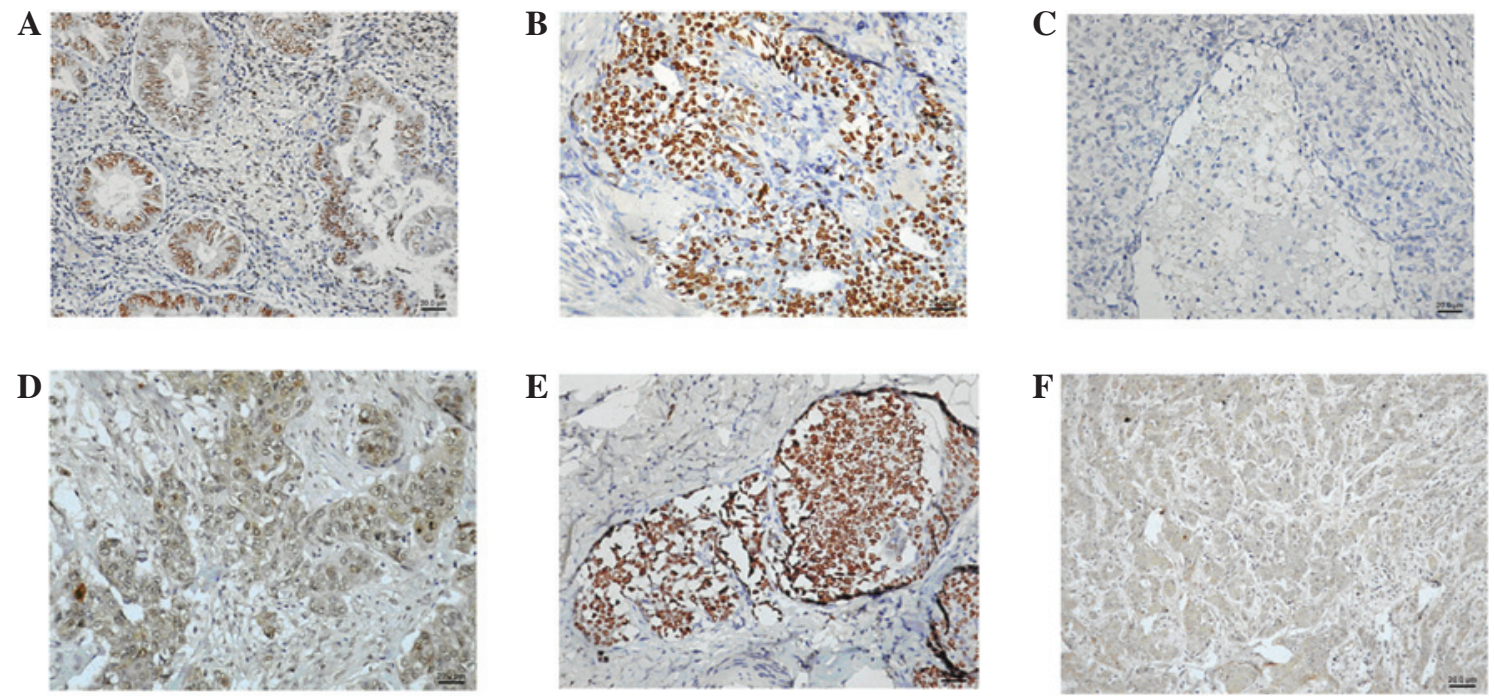

Figure 1. Immunohistochemical results of estrogen receptor $\alpha$ phosphorylation at serine 118 (pER $\alpha$-S118) and 167 (pER $\alpha$-S167) in patients with primary breast cancer. (A) pER $\alpha-S 118$ (+); (B) pER $\alpha-S 118$ (+++); (C) pER $\alpha-S 118$ (-); (D) pER $\alpha-S 167$ (+); (E) pER $\alpha-S 167$ (+++); and (F) pER $\alpha-S 167$ (-). Magnification, x200. -, negative; + , weakly positive; +++ , strongly positive.

Ser 167, and also predicted the resistance of breast cancer to tamoxifen.

\section{Materials and methods}

Patients and breast cancer tissues. Breast tumor specimens from 104 female patients with invasive breast carcinoma, who had registered at The Affiliated Cancer Hospital of Shantou University Medical College between January 2001 and December 2007, were included in the present study. The study protocol was approved by the institutional review board of Shantou University Medical College (Guangdong, China). Written informed consent was obtained from all patients. All patients had undergone surgical treatments for primary breast cancer (either mastectomy or lumpectomy), and all primary tumors were ER $\alpha$-positive, as defined by immunohistochemical staining. The samples were selected from a continuous series of invasive carcinoma tissues. Following surgery, 78 patients received systemic adjuvant chemotherapy and 47 received radiotherapy. All patients received $10 \mathrm{mg}$ tamoxifen twice a day as an endocrine therapy, either until the disease had progressed or for five years, following the previously mentioned treatments. The follow-up time ranged from 50-121 months.

Immunohistochemical analysis. A 4- $\mu \mathrm{m}$ section of each submitted paraffin block was first stained with hematoxylin and eosin to verify that an adequate number of invasive carcinoma cells were present. Serial sections $(4 \mu \mathrm{m})$ were prepared from selected blocks and float-mounted onto adhesive-coated glass slides. In order to stain for $\mathrm{pER} \alpha$-Ser118/167, the slides were oven-boiled in a citrate buffer ( $\mathrm{pH}$ 6.0) for $20 \mathrm{~min}$, cooled at room temperature for $20 \mathrm{~min}$ and then placed into methanol containing $3 \% \mathrm{H}_{2} \mathrm{O}_{2}$ for $5 \mathrm{~min}$ to inactivate the endogenous peroxidase. This was followed by $10 \mathrm{~min}$ of incubation with a serum-free protein block solution (DakoCytomation, Glostrup, Denmark). The slides were subsequently washed three times and incubated with 1:100 diluted anti-rabbit polyclonal $\mathrm{pER} \alpha$ -
S118/167 antibodies (Cell Signaling Technology, Inc., Boston, MA, USA). Following this, the slides were placed in a moisturized chamber at $4^{\circ} \mathrm{C}$ for $20 \mathrm{~h}$. The slides were then washed, incubated with the anti-rabbit DakoCytomation Envision+ system for $30 \mathrm{~min}$ at $4^{\circ} \mathrm{C}$, visualized with 3,3'-diaminobenzidine hydrochloride in phosphate buffer containing $0.03 \%$ $\mathrm{H}_{2} \mathrm{O}_{2}$ and counterstained with hematoxylin. All washing steps were performed in phosphate-buffered saline solution with $0.5 \%$ bovine serum albumin. The staining intensity was evaluated on three separate biopsies for each tumor and was scored by two trained histopathologists, using the modified McCarty's H-scoring system (6). This utilized the percentage of positive cells and the intensity of staining to provide a total score, varying from $0-300$. The staining was designated as negative (-; H-score, $<50)$, weakly positive (+; H-score, 51-100), moderately positive (++; H-score, 101-200) or strongly positive (+++; H-score, 201-300) As the proportion of positive staining was moderate, the cutoff point for positive staining was set to $\geq 1 \%$ in the statistical analysis. $\mathrm{pER} \alpha-\mathrm{S} 118 / 167$ was occasionally visible in the cytoplasm, but only nuclear staining was graded.

Statistical analysis. The $\chi^{2}$ test was used to compare the expression of $\mathrm{pER} \alpha-\mathrm{S} 118 / 167$ with the clinicopathological characteristics. The student's t-test was used to compare the survival time between the uni- and coexpression of $\mathrm{pER} \alpha-\mathrm{S} 118 / 167$. An estimation of patient survival was performed using the Cox regression method, and a KaplanMeier curve was used to assess the survival differences between the pER $\alpha$-S118-postive and -negative patients, as well as between the pER $\alpha$-S167-positive and -negative samples. $\mathrm{P}<0.05$ was considered to indicate a statistically significant difference.

\section{Results}

Correlation between $p E R \alpha-S 118$ or $p E R \alpha-S 167$ expression and clinicopathological factors in primary breast tumors. 
Table I. Correlation between clinicopathological factors, DFS and OS in 104 primary breast tumors of luminal type.

\begin{tabular}{|c|c|c|c|c|c|}
\hline \multirow[b]{2}{*}{ Clinicopathological factors } & \multirow[b]{2}{*}{ No. of cases } & \multicolumn{2}{|c|}{ DFS } & \multicolumn{2}{|c|}{ OS } \\
\hline & & Wald statistic & P-value & Wald statistic & P-value \\
\hline Age (years) & & 4.786 & 0.680 & 5.865 & 0.566 \\
\hline$\leq 50$ & 48 & & & & \\
\hline$>50$ & 56 & & & & \\
\hline Menopausal status & & 2.735 & 0.541 & 3.100 & 0.432 \\
\hline Premenopausal & 59 & & & & \\
\hline Postmenopausal & 45 & & & & \\
\hline $\mathrm{T}$ & & 4.313 & 0.040 & 2.495 & 0.023 \\
\hline $\mathrm{T} 1$ & 19 & & & & \\
\hline $\mathrm{T} 2$ & 57 & & & & \\
\hline $\mathrm{T} 3$ & 24 & & & & \\
\hline $\mathrm{T} 4$ & 4 & & & & \\
\hline $\mathrm{N}$ & & 17.467 & 0.002 & 23.220 & 0.000 \\
\hline No & 48 & & & & \\
\hline $\mathrm{N} 1$ & 27 & & & & \\
\hline $\mathrm{N} 2$ & 17 & & & & \\
\hline $\mathrm{N} 3$ & 12 & & & & \\
\hline ER & & 3.133 & 0.372 & 4.319 & 0.229 \\
\hline - & 3 & & & & \\
\hline+ & 10 & & & & \\
\hline++ & 25 & & & & \\
\hline+++ & 66 & & & & \\
\hline PR & & 1.427 & 0.699 & 2.051 & 0.562 \\
\hline- & 23 & & & & \\
\hline+ & 23 & & & & \\
\hline++ & 23 & & & & \\
\hline+++ & 35 & & & & \\
\hline HER-2 & & 4.174 & 0.041 & 2.598 & 0.107 \\
\hline Negative & 47 & & & & \\
\hline Postive & 57 & & & & \\
\hline p-ER $\alpha-S 118$ & & 1.686 & 0.194 & 2.882 & 0.090 \\
\hline Negative & 78 & & & & \\
\hline Postive & 26 & & & & \\
\hline p-ER $\alpha-S 167$ & & 1.738 & 0.187 & 0.989 & 0.320 \\
\hline Negative & 76 & & & & \\
\hline Postive & 28 & & & & \\
\hline
\end{tabular}

DFS, disease-free survival; OS, overall survival; T, tumor size; N, axillary lymph node staging; ER, estrogen receptor; PR, progesterone receptor; HER-2, human epidermal growth factor receptor 2; p-ER $\alpha$-S118, estrogen receptor $\alpha$ phosphorylation at serine 118; p-ER $\alpha-S 167$, estrogen receptor $\alpha$ phosphorylation at serine 167 ; -, negative; +, weakly positive; ++, moderately positive; +++, strongly positive.

$\mathrm{pER} \alpha-\mathrm{S} 118$ and/or pER $\alpha$-S167 were immunohistochemically detected in a total of 104 primary invasive breast carcinomas, as demonstrated in Fig. 1. A total of 25.0\% (26/104) of carcinomas were positive for pER $\alpha-\mathrm{S} 118$ (Figs. 1A-C), whereas $26.9 \%$ (28/104) were positive for pER $\alpha-S 167$ (Figs. 1D-F). All graded phosphorylation staining occurred in the nucleus.

The DFS and OS values of 104 primary breast cancer patients are shown in Fig 2, whilst the correlation between the clinicopathological factors and the survival of the patients is shown in Table I. The factors affecting the DFS and OS were the tumor size and axillary lymph node staging. DFS was also shown to be affected by the HER-2 status $(\mathrm{P}=0.041)$. No correlation was observed between $\mathrm{pER} \alpha-\mathrm{S} 118 / 167$ and survival time.

The immunohistochemistry (IHC) scores for $\mathrm{pER} \alpha-\mathrm{S} 118$ and $\mathrm{pER} \alpha-\mathrm{S} 167$ were correlated with various clinicopathological factors. The clinicopathological factor distribution between the positive and negative expression of $\mathrm{pER} \alpha-\mathrm{S} 118$ and pER $\alpha-\mathrm{S} 167$ is demonstrated in Table II. A significant 
Table II. Correlation between expression of $\mathrm{pER} \alpha$-Ser118 and $\mathrm{pER} \alpha$-Ser167 and the clinicopathological factors in primary breast tumors.

\begin{tabular}{|c|c|c|c|c|c|c|c|c|}
\hline \multirow{2}{*}{$\begin{array}{l}\text { Clinicopathological } \\
\text { factors }\end{array}$} & \multicolumn{4}{|c|}{ pER $\alpha-\operatorname{Ser} 118$} & \multicolumn{4}{|c|}{ pER $\alpha-\operatorname{Ser} 167$} \\
\hline &,$+ \mathrm{n}(\%)$ &,$- \mathrm{n}(\%)$ & $\chi^{2}$ & P-value &,$+ \mathrm{n}(\%)$ &,$- \mathrm{n}(\%)$ & $\chi^{2}$ & P-value \\
\hline \multicolumn{9}{|l|}{$\mathrm{T}$} \\
\hline $\mathrm{T} 1$ and $\mathrm{T} 2$ & $22(28.57)$ & $55(71.43)$ & 2.02 & 0.16 & $23(30.26)$ & $53(69.74)$ & 1.88 & 0.17 \\
\hline $\mathrm{T} 3$ and $\mathrm{T} 4$ & $4(14.81)$ & $23(85.19)$ & & & $5(17.86)$ & $23(82.14)$ & & \\
\hline \multicolumn{9}{|l|}{$\mathrm{N}$} \\
\hline N0 and N1 & $19(25.33)$ & $56(74.67)$ & 0.02 & 0.90 & $17(22.67)$ & $58(77.33)$ & 2.80 & 0.09 \\
\hline $\mathrm{N} 2$ and N3 & 7 (24.14) & $22(75.86)$ & & & $11(37.93)$ & $18(62.07)$ & & \\
\hline \multicolumn{9}{|l|}{ PR } \\
\hline Positive & $20(24.69)$ & $61(75.31)$ & 0.95 & 0.33 & $22(27.16)$ & $59(72.84)$ & 1.56 & 0.21 \\
\hline Negative & $6(26.09)$ & $17(73.91)$ & & & $6(26.09)$ & $17(73.91)$ & & \\
\hline \multicolumn{9}{|l|}{ HER-2 } \\
\hline Positive & $20(35.09)$ & 37 (64.91) & 6.85 & 0.01 & $15(26.32)$ & $42(73.68)$ & 0.23 & 0.63 \\
\hline Negative & $6(12.77)$ & $41(87.23)$ & & & $13(27.66)$ & $34(72.34)$ & & \\
\hline \multicolumn{9}{|l|}{ Age (years) } \\
\hline$<50$ & $10(20.83)$ & 38 (79.17) & 0.83 & 0.36 & $15(31.25)$ & $33(68.75)$ & 0.85 & 0.36 \\
\hline$\geq 50$ & $16(28.57)$ & $40(71.43)$ & & & $13(23.21)$ & 43 (76.79) & & \\
\hline \multicolumn{9}{|l|}{ Menopausal status } \\
\hline Premenopausal & $14(23.73)$ & 45 (76.27) & 0.12 & 0.73 & $12(20.34)$ & 47 (79.66) & 3.00 & 0.08 \\
\hline Postmenopausal & $12(26.67)$ & $33(73.33)$ & & & $16(35.56)$ & $29(64.44)$ & & \\
\hline
\end{tabular}

pER $\alpha$-S118, estrogen receptor $\alpha$ phosphorylation at serine 118; pER $\alpha$-S167, estrogen receptor $\alpha$ phosphorylation at serine 167; T, tumor size; $\mathrm{N}$, axillary lymph node staging; PR, progesterone receptor; Age, age at diagnosis; HER-2, human epidermal growth factor receptor 2.
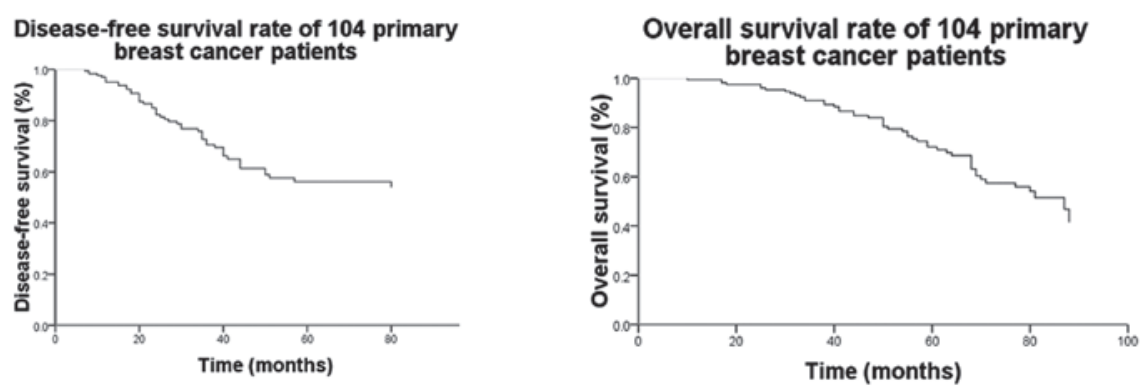

Figure 2. Kaplan-Meier graphs for the disease-free and overall survival rates of 104 primary breast cancer patients undergoing tamoxifen therapy.

positive correlation was found between $\mathrm{pER} \alpha-\mathrm{S} 118$ expression and HER-2 status, as determined by IHC $\left(\chi^{2}=6.85\right.$, $\mathrm{P}=0.01)$. Among the $26 \mathrm{pER} \alpha-\mathrm{S} 118$-positive patients, 20 were HER-2 positive, while out of the $76 \mathrm{pER} \alpha-\mathrm{S} 118$-negative patients, 37 were HER-2 positive. In contrast, no correlation was identified between pER $\alpha-S 167$ and the HER-2 status $\left(\chi^{2}=0.23, \mathrm{P}=0.63\right)$. There was also no significant difference observed between pER $\alpha-\mathrm{S} 118 / \mathrm{pER} \alpha-\mathrm{S} 167$ and the tumor size $\left(\chi^{2}=2.02, \mathrm{P}=0.16\right.$ and $\chi^{2}=1.88, \mathrm{P}=0.17$, respectively) or lymph node status $\left(\chi^{2}=0.02, \mathrm{P}=0.90\right.$ and $\mathrm{P}=0.09, \chi^{2}=2.80$, respectively). In addition, there was no correlation between $\mathrm{pER} \alpha-\mathrm{S} 118 / 167$ and the progesterone receptor status $\left(\chi^{2}=0.95\right.$, $\mathrm{P}=0.33$ and $\chi^{2}=1.56, \mathrm{P}=0.21$, respectively), the age at diagnosis $\left(\chi^{2}=0.83, \mathrm{P}=0.36\right.$ and $\chi^{2}=0.85, \mathrm{P}=0.36$, respectively) and the menopausal status $\left(\chi^{2}=0.12, P=0.73\right.$ and $\chi^{2}=3.00$, $\mathrm{P}=0.08$, respectively).

pER $\alpha-S 118$ may predict the resistance of breast cancer to tamoxifen. The primary aim of the present study was to determine whether $\mathrm{pER} \alpha-\mathrm{S} 118$ or $\mathrm{pER} \alpha-\mathrm{S} 167$ were related to the clinical outcome in patients treated with tamoxifen. pER $\alpha-\mathrm{S} 118 / \mathrm{S} 167$ was determined to be a binary factor (positive where detectable nuclear staining was present and negative where detectable nuclear staining was absent) and a Kaplan-Meier survival analysis was performed since fewer than one-third of the samples exhibited positive staining for either $\mathrm{pER} \alpha-\mathrm{S} 118$ or $\mathrm{pER} \alpha-\mathrm{S} 167$. Kaplan-Meier plots of DFS and OS are shown in Fig. 3. Those patients whose primary 
Table III. Correlation between pER $\alpha-S 118 / 167$ and the DFS and OS times.

\begin{tabular}{lcccc}
\hline & Number & DFS (months) & P-value & OS (months) \\
\hline pER $\alpha-S 118(+)$ & 19 & $39.68 \pm 6.11$ & 0.79 & $53.42 \pm 4.75$ \\
pER $\alpha-S 167(+)$ & 21 & $64.90 \pm 7.46$ & 0.26 & $76.62 \pm 5.61$ \\
Both (+) & 7 & $41.71 \pm 10.00$ & & $53.86 \pm 7.68$ \\
Both (-) & 57 & $55.32 \pm 3.67$ & & $63.65 \pm 2.95$ \\
\hline
\end{tabular}

DFS and OS are presented as mean \pm SD. P-value; comparison with the Both (+) group. DFS, disease-free survival; OS, overall survival; pER $\alpha-S 118$, estrogen receptor $\alpha$ phosphorylation at serine 118; pER $\alpha-S 167$, estrogen receptor $\alpha$ phosphorylation at serine 167; (+), positive expression; (-) negative expression.

A

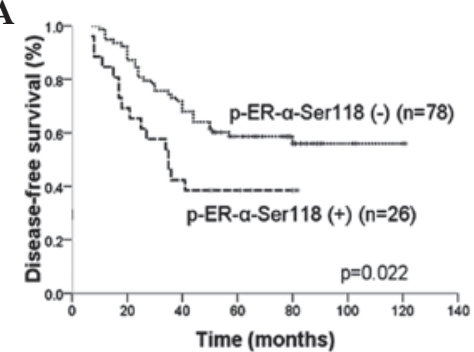

C

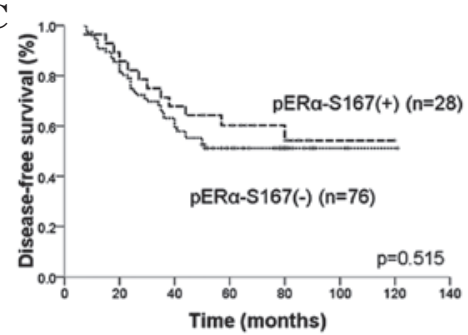

B

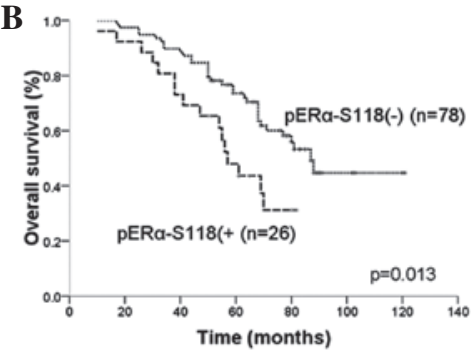

D

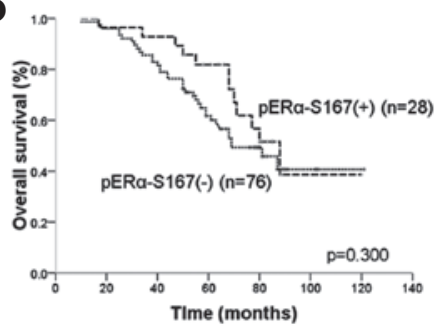

Figure 3. Kaplan-Meier graphs for the effect of the phosphorylation of estrogen receptor $\alpha$ at serine 118 (pER $\alpha$-S118) and 167 (pER $\alpha$-S167) on (A,C) diseasefree and (B,D) overall survival. (A) $\chi^{2}=5.218$ and $\mathrm{P}=0.022$; (B) $\chi^{2}=6.216$ and $\mathrm{P}=0.013$; (C) $\chi^{2}=0.424$ and $\mathrm{P}=0.515$; and (D) $\chi^{2}=1.075$ and $\mathrm{P}=0.300$.

tumors expressed $\mathrm{pER} \alpha-\mathrm{S} 118$ had a shorter DFS and OS than those whose tumors were $\mathrm{pER} \alpha$-S118-negative $(\mathrm{P}=0.022$ and $\mathrm{P}=0.013$, respectively; Figs. $3 \mathrm{~A}$ and $\mathrm{B})$. Although there was an apparent correlation between $\mathrm{pER} \alpha-\operatorname{Ser} 167$ and OS (Figs. 3C and D), the results were not significant $(\mathrm{P}=0.515$ and $\mathrm{P}=0.300$, respectively; Fig. 3). This was most likely due to the small number of events (fatalities) occurring in the present study.

Among the 104 patients, there were seven samples expressing positive staining for $\mathrm{pER} \alpha-\mathrm{S} 118$ and $\mathrm{pER} \alpha-\mathrm{S} 167$. A comparison between these seven samples and those samples exhibiting positive staining for either $\mathrm{pER} \alpha-\mathrm{S} 118$ or $\mathrm{pER} \alpha-\mathrm{S} 167$ indicated that the uni-/coexpression of $\mathrm{pER} \alpha-\mathrm{S} 118 / \mathrm{pER} \alpha-\mathrm{S} 167$ had no effect on DFS and OS (Table III).

\section{Discussion}

Although third generation aromatase inhibitors exhibit certain advantages over tamoxifen in postmenopausal breast cancer patients, tamoxifen remains the first-line treatment for numerous ER-positive breast cancer patients. Approximately one-third of ER-positive tumors will gradually develop resistance to endocrine therapy and, in particular, to tamoxifen treatment (2). The determination of the prognostic factor(s) for breast cancer patients treated with tamoxifen may aid in the optimization of the therapeutic strategy and also in overcoming the resistance to endocrine therapy.

It has been demonstrated that ER- $\alpha$ may be phosphorylated on multiple amino acid residues (4). In general, phosphorylation in the activation function-1 (AF1) domain of ER $\alpha$ appears to recruit coactivators, resulting in enhanced ER $\alpha$-mediated transcription, and also affect the cellular response to tamoxifen (7). In the present study, the nuclear expression of $\mathrm{pER} \alpha-\mathrm{S} 118$ and $\mathrm{pER} \alpha-\mathrm{S} 167$ was detected by IHC and the correlation with tamoxifen responsiveness was also analyzed.

It was identified that $\mathrm{pER} \alpha-\mathrm{S} 118$ and $\mathrm{pER} \alpha-\mathrm{S} 167$ were expressed in a proportion of tamoxifen-resistant breast tumors, and that their expression did not exhibit any correlation with the age, menopausal status, TNM (tumor size, axillary lymph node staging) stage or ER and PR status of the patient. However, $\mathrm{pER} \alpha-\mathrm{S} 118$, but not $\mathrm{pER} \alpha-\mathrm{S} 167$, was significantly correlated with the expression level of HER-2 and with a shorter survival time in breast cancer patients. The HER-2 levels are amplified in $20 \%$ of breast cancer patients and HER-2 overexpression is associated with a poorer prognosis (8). The HER-2 gene is located on the $17 \mathrm{q}$ chromosome and encodes a transmembrane tyrosine growth receptor that produces a protein receptor on 
the cell membrane with a molecular weight of $\sim 185 \mathrm{kDa}(9)$. It has been confirmed that HER-2 may be correlated with the advanced progression and poorer prognosis of breast cancer. Cittelly et al demonstrated that breast cancer overexpressing HER-2 exhibited resistance to tamoxifen through the upregulation of B-cell CLL/lymphoma 2 (BCL-2) and the suppression of miR-15a/16 induced by tamoxifen (10). Yamashita et al indicated that $\mathrm{pER} \alpha-\mathrm{S} 118$ was positively and significantly correlated with HER-2 and that it was amplified in the AIB1 gene (11). Certain studies have revealed that ER $\alpha$ may be phosphorylated at Ser 118 by ERK-MAPK $(12,13)$. ERK-MAPK is a downstream kinase of HER-2 (14), and thus the HER2MAPK-AIB1-pER $\alpha$-S118 pathway may form a kinase cascade that leads to a poorer prognosis when the pathway is activated. Further studies into this mechanism are required.

Most notably, the present analysis has demonstrated that $\mathrm{pER} \alpha-\mathrm{S} 118$ is correlated with poorer survival. $\mathrm{pER} \alpha-\mathrm{S} 118$ was associated with a shorter DFS and OS $(\mathrm{P}=0.022$ and $\mathrm{P}=0.013$, respectively), however, there was no statistically significant correlation between $\mathrm{pER} \alpha-\mathrm{S} 167$ and the DFS or OS of the breast cancer patients $(\mathrm{P}=0.515$ and $\mathrm{P}=0.300$, respectively). Previous studies in vivo and in vitro remain divided over whether $\mathrm{pER} \alpha-\mathrm{S} 167$ or $\mathrm{pER} \alpha-\mathrm{S} 118$ have an impact on tamoxifen resistance (11,15-18). Sarwar et al demonstrated that Ser 118 phosphorylation was elevated in tumor biopsies taken from patients who had relapsed following tamoxifen treatment (19). Yamashita et al revealed that a higher expression of $\mathrm{pER} \alpha-\mathrm{S} 118$ was a predictor of poorer survival, which is consistent with the present study (11). By contrast, Murphy et al analyzed pER $\alpha-\mathrm{S} 118$ by IHC in 117 breast cancer tissues and demonstrated that it was a marker of improved prognosis in patients treated with tamoxifen (20). However, in the study by Murphy et al, the determination of ER $\alpha$-positive tumors was analyzed by ligand binding assay, not by IHC, and their patient inclusion criteria comprised axillary lymph node-negative, and not only ER $\alpha$-positive, tumors. Other studies have also demonstrated that $\mathrm{pER} \alpha$ S167 has an effect on the survival of breast cancer patients. Yamashita et al indicated that a higher expression of $\mathrm{pER} \alpha$ S167 was correlated with improved survival in ER-positive breast cancers (11). However, Guo et al demonstrated that $\mathrm{pER} \alpha-$ Ser167 was phosphorylated by inhibitor of kappa B kinase- $\varepsilon(\mathrm{IKK} \varepsilon)$ in vitro and in vivo, leading to the upregulation of cyclin D1 and resulting in tamoxifen resistance (16). In the present study, however, we failed to demonstrate any correlation between $\mathrm{pER} \alpha-\mathrm{S} 167$ and tamoxifen resistance. There may be several reasons for these discrepancies; for example, there were varying detection methods and cutoff points for diagnosis, and so uniform standards of detection should be discussed and investigated in the future. In addition, there were differing inclusion criteria for the samples. Although certain studies took biopsies $(17,21)$, a number of studies used samples following surgery $(11,17,20)$. Moreover, there were differences in the treatment that the patients received following surgery. The patients in the present study received chemotherapy and/or radiation according to National Comprehensive Cancer Network (NCCN) guidelines. However, in the study by Yamashita et al, patients with positive axillary lymph nodes did not receive radiation (11). It is therefore difficult to estimate the true effect of tamoxifen therapy in post-operative adjuvant therapy. Furthermore, the effect of ethnicity remains unclear.

Combinatorial regulation has been discussed in numerous biochemical phenomena, including the cophosphorylation of various sites in ER $\alpha$ (22). In the present study, the coexpression of pER $\alpha-S 118$ and $\mathrm{pER} \alpha-\mathrm{S} 167$ was detected in seven out of the 104 samples (6.73\%). The DFS and OS of these patients were similar to that of patients solely expressing $\mathrm{pER} \alpha-\mathrm{S} 118$ ( $\mathrm{P}=0.79$ and 0.87 , respectively). The OS was significantly different from that of patients solely expressing pER $\alpha-\mathrm{S} 167$ $(\mathrm{P}=0.05)$, suggesting that $\mathrm{pER} \alpha-\mathrm{S} 167$ may not be a significant predictor for breast cancer responsiveness to tamoxifen. However, the DFS was not significantly different from that of patients solely expressing pER $\alpha-\mathrm{S} 167(\mathrm{P}=0.26)$. As there were only seven cases of coexpression in the present study, a larger sample size is required in future studies.

In conclusion, the present study found that adjuvant tamoxifen-treated breast cancer patients who have primary tumors expressing pER $\alpha-\mathrm{S} 118$ have a shorter DFS and OS. The data suggest that $\mathrm{pER} \alpha-\mathrm{S} 118$ may be an indicator for the resistance of breast cancer to tamoxifen.

\section{Acknowledgements}

This study was partly funded by the National Natural Science Foundation Committee (grant nos. 30973377 and 31271068) and the Medical Scientific Research Foundation of Guangdong Province (grant no. B2010225).

\section{References}

1. Lim E, Metzger-Filho O and Winer EP: The natural history of hormone receptor-positive breast cancer. Oncology (Williston Park) 26: 688-694, 696, 2012.

2. Murphy LC, Seekallu SV and Watson PH: Clinical significance of estrogen receptor phosphorylation. Endocr Relat Cancer 18: R1-R14, 2011.

3. Becker MA, Ibrahim YH, Cui X, Lee AV and Yee D: The IGF pathway regulates ER $\alpha$ through a S6K1-dependent mechanism in breast cancer cells. Mol Endocrinol 25: 516-528, 2011.

4. de Leeuw R, Neefjes J and Michalides R: A role for estrogen receptor phosphorylation in the resistance to tamoxifen. Int J Breast Cancer 2011: 232435, 2011.

5. Green KA and Carroll JS: Oestrogen-receptor-mediated transcription and the influence of co-factors and chromatin state. Nat Rev Cancer 7: 713-722, 2007

6. McCarty KS Jr, Miller LS, Cox EB, Konrath J and McCarty KS Sr: Estrogen receptor analyses. Correlation of biochemical and immunohistochemical methods using monoclonal antireceptor antibodies. Arch Pathol Lab Med 109: 716-721, 1985.

7. Riggins RB, Schrecengost RS, Guerrero MS and Bouton AH: Pathways to tamoxifen resistance. Cancer Lett 256: 1-24, 2007.

8. Thibault C, Khodari W, Lequoy M, Gligorov J and Belkacémi Y: HER2 status for prognosis and prediction of treatment efficacy in adenocarcinomas: A review. Crit Rev Oncol Hematol 13: Apr 5, 2013 (Epub ahead of print).

9. Slamon DJ, Clark GM, Wong SG, Levin WJ, Ullrich A and McGuire WL: Human breast cancer: correlation of relapse and survival with amplification of the HER-2/neu oncogene. Science 235: 177-182, 1987.

10. Cittelly DM, Das PM, Salvo VA, Fonseca JP, Burow ME and Jones FE: Oncogenic HER $2 \Delta 16$ suppresses miR-15a/16 and deregulates BCL-2 to promote endocrine resistance of breast tumors. Carcinogenesis 31: 2049-2057, 2010.

11. Yamashita H, Nishio M, Toyama T, et al: Low phosphorylation of estrogen receptor alpha (ERalpha) serine 118 and high phosphorylation of ERalpha serine 167 improve survival in ER-positive breast cancer. Endocr Relat Cancer 15: 755-763, 2008. 
12. Métivier R, Gay FA, Hübner MR, et al: Formation of an hER alpha-COUP-TFI complex enhances hER alpha AF-1 through Ser118 phosphorylation by MAPK. EMBO J 21: 3443-3453, 2002.

13. Zhu C, Qi X, Chen Y, Sun B, Dai Y and Gu Y: PI3K/Akt and MAPK/ERK1/2 signaling pathways are involved in IGF-1-induced VEGF-C upregulation in breast cancer. J Cancer Res Clin Oncol 137: 1587-1594, 2011.

14. Tai W, Qin B and Cheng K: Inhibition of breast cancer cell growth and invasiveness by dual silencing of HER-2 and VEGF. Mol Pharm 7: 543-556, 2010.

15. Mintz PJ, Habib NA, Jones LJ, et al: The phosphorylated membrane estrogen receptor and cytoplasmic signaling and apoptosis proteins in human breast cancer.Cancer 113: 1489-1495, 2008.

16. Guo JP, Shu SK, Esposito NN, Coppola D, Koomen JM and Cheng JQ: IKKepsilon phosphorylation of estrogen receptor alpha Ser-167 and contribution to tamoxifen resistance in breast cancer. J Biol Chem 285: 3676-3684, 2010.

17. Motomura K, Ishitobi M, Komoike Y, et al: Expression of estrogen receptor beta and phosphorylation of estrogen receptor alpha serine 167 correlate with progression-free survival in patients with metastatic breast cancer treated with aromatase inhibitors. Oncology 79: 55-61, 2010.
18. Murphy LC, Skliris GP, Rowan BG, et al: The relevance of phosphorylated forms of estrogen receptor in human breast cancer in vivo. J Steroid Biochem Mol Biol 114: 90-95, 2009.

19. Sarwar N, Kim JS, Jiang J, et al: Phosphorylation of ERalpha at serine 118 in primary breast cancer and in tamoxifen-resistant tumours is indicative of a complex role for ERalpha phosphorylation in breast cancer progression. Endocr Relat Cancer 13: 851-861, 2006.

20. Murphy LC, Niu Y, Snell L and Watson P: Phospho-serine-118 estrogen receptor-alpha expression is associated with better disease outcome in women treated with tamoxifen. Clin Cancer Res 10: 5902-5906, 2004.

21. Murphy LC, Weitsman GE, Skliris GP, et al: Potential role of estrogen receptor alpha (ERalpha) phosphorylated at Serine118 in human breast cancer in vivo. J Steroid Biochem Mol Biol 102: 139-146, 2006.

22. Bhardwaj N, Carson MB, Abyzov A, Yan KK, Lu H and Gerstein MB. Analysis of combinatorial regulation: scaling of partnerships between regulators with the number of governed targets. PLoS Comput Biol 6: e1000755, 2010. 